\section{Dr. Tebib replies}

\section{To the Editor:}

I thank Dr. Toms and colleagues for bringing more discussion to the difficult problem of overassessing disease activity in RA patients also affected with fibromyalgia (FM) when a composite index is used such as the 28-joint Disease Activity Score (DAS-28).

Their experience presents a lot of similarities to ours, such as the FM prevalence in a rheumatoid arthritis (RA) outpatient population that is estimated around $25 \%$ in both of our experiences, which stresses the high frequency of this clinical situation. Likewise, our results of the assessment in terms of tender, swollen joints and DAS are remarkably close and these results might draw attention when preparing a trial that uses a composite index as the primary endpoint.

However, some differences deserve to be analyzed because they could raise more interesting questions on this difficult problem.

The first is the relatively unexpected high frequency of men affected by FM (almost 20\%) in their RA population. This allowed our colleagues to consider their whole male/female concomitant fibromyalgia (RAF) population for comparison with their RA control. We could not do so because of the large difference in sex ratio between our patients with RA and patients with RAF, which led us to limit our analysis only to the RAF and RA female population. Toms, et al's unusual result ${ }^{1}$ deserves to be confirmed by other studies when associated with RA because this could shed new light on the discussion of the relationship between the 2 diseases. Indeed, with the similar profile of FM in RA and in an FM control population, we offer the possibility that the 2 diseases were just associated and not linked, while considering the stress induced by RA to explain the higher frequency of FM in this population, maybe by eliciting an FM background ${ }^{2}$. If their results are confirmed, the discrepancy in terms of sex ratio in RAF compared to control population could make more questionable our hypothesis of a "simple" association. On the other hand, the relative increase of male prevalence could then explain the absence of social status difference between their RA and RAF population, contrary to our results and some others ${ }^{1,3}$. Indeed, more severe psychological distress in women affected by arthritis compared with the male arthritis population is usually pointed out ${ }^{4}$ and then associated with a rather lower sociocultural profile ${ }^{1}$, similar to those observed in our study (identical to those of an FM female control population we allowed to participate).
The second point of interest is that Dr. Toms used the DAS-28 and the Clinical Disease Activity Index and the Simplified Disease Activity Index, which allow the physician to give an opinion on the patient's state. Interestingly, the professional opinion was also significantly higher in the RAF population, leading to the conclusion that either the authors don't consider the difference between the 2 populations to be important (even though they actually draw attention to this point), or they take more into account the pain distress of the patients with RAF rather than the objective joints state. This opens again the discussion of the treatment of the RAF population in terms of inefficient disease-modifying antirheumatic drugs (DMARD) escalation or of a beneficial management of the pain. In any case, this demonstrates again the very difficult problem of assessment in patients with RAF.

JACQUES TEBIB, MD, PhD, CHLS, Service du Rhumatologie, 14 Ch du Petit Revoyet, Pierre Benite 69495, France. Address correspondence to Dr. Tebib; E-mail: tebib@aol.com

\section{REFERENCES}

1. Wolfe F, Ross K, Anderson J, Russell J, Herbert L. The prevalence and characteristics of fibromyalgia in the general population. Arthritis Rheum 1995,38:19-28.

2. Coury F, Rossat A, Tebib A, Letroublon MC, Gagnard A, Fantino $\mathrm{B}$, et al. Rheumatoid arthritis and fibromyalgia: A frequent unrelated association complicating disease management. J Rheumatol 2009;36:58-62.

3. Assumpção A, Cavalcante AB, Capela CE, Sauer JF, Chalot SD, Pereira CA, et al. Prevalence of fibromyalgia in a low socioeconomic status population. BMC Musculoskelet Disord 2009; 10:64.

4. Shih M, Hootman JM, Strine TW, Chapman DP, Brady TJ. Serious psychological distress in U.S. adults with arthritis. J Gen Intern Med 2006;21:1160-6.

J Rheumatol 2010;37:2; doi:10.3899/jrheum.090976 